\title{
The optimisation of the single surface mount device placement machine in printed circuit board assembly: a survey
}

\author{
Masri Ayob $^{\mathrm{a}}$ and Graham Kendall ${ }^{\mathrm{b} *}$ \\ ${ }^{a}$ Department of Computer Science, Faculty of Information Science and Technology, University Kebangsaan Malaysia, 43600 \\ Bangi, Selangor, Malaysia; ${ }^{b}$ ASAP Research Group, School of Computer Science, The University of Nottingham, \\ Nottingham NG8 1BB, UK
}

(Received 25 July 2007; final version received 28 May 2008)

\begin{abstract}
This article surveys research on the single surface mount device (SMD) placement machine optimisation problem. We classify the optimisation problem into five sub-problems: feeder setup, component placement sequencing, nozzle optimisation, component retrieval plan and motion control; and analyse issues relevant to each of these. One of the aims of this article is to provide guidance to other researchers and gain a deeper understanding of the various optimisation issues that arise in this domain. This could lead to the design of improved heuristics, which are more appropriate to the real-world scheduling problem of the SMD placement machine.
\end{abstract}

Keywords: scheduling; heuristic; printed circuit board assembly; component placement sequencing; feeder setup

\section{Introduction}

Over the last 20 years, printed circuit board (PCB) production has evolved from a labour-intensive activity to a highly automated activity (Crama, Flippo, Klundert and Spieksma 1997). The introduction of surface mount technology (SMT) has almost replaced pin-through-hole technology in PCB assembly and has enabled the production of high density (allowing many components to be placed onto a PCB in a small area) PCB's (Jeevan, Parthiban, Seetharamu, Azid and Quadir 2002). However, pin-through-hole technology is still preferred for some applications which use highvoltage components, or for applications subject to environmental stresses such as vibration. To be more competitive in today's global marketplace, PCB assembly manufacturers are striving to respond to emerging trends including high quality, low-cost and just in-time delivery. Therefore, in order to enhance their competitiveness, many PCB assembly manufacturers are developing computer integrated manufacturing systems that are capable of producing an effective planning, scheduling and control procedure. Moreover, the demand of automating PCB assembly is increasing with the miniaturisation of component designs and the increasing density of components on the PCB (Moyer and Gupta 1996a and b).

Tirpak (2000) asserts that SMT assembly involves three operations: solder paste, component placement and solder reflow. Surface mount device (SMD) placement machines, which cost between $\$ 300,000$ and $\$ 1,000,000$, are often a bottleneck in the assembly line (Moyer and Gupta 1997; Tirpak, Nelson and Aswani 2000; Csaszar, Nelson, Rajbhandari and Tirpak 2000a). It would obviously be beneficial if more effective use could be made of this expensive resource.

Once a PCB has been loaded into the machine, a fiducial marks (two to four points, located near the corners of the PCB) operation identifies the position and orientation of the PCB (Magyar, Johnsson and Nevalainen 1999). Once the PCB is secured, the components are placed onto the PCB. The software which guides the placement operation is usually supplied by the machine vendor and is often not very efficient (Shih, Srihari and Adriance 1996). Indeed, Magyar et al. (1999) argued that until now, PCB machine vendors and software companies have not been capable of solving even a single machine optimisation problem efficiently. Once all available components have been placed (some may be missing due to component run outs) the PCB is unloaded from the machine before undergoing a soldering process to adhere components to the PCB (known as solder reflow) (Leu, Wong and Ji 1993).

Due to a lack of standardisation among SMD placement machines, the optimisation of the pick-andplace operations is largely influenced by the constraints of a given machine and the production environment under which the machine is installed (Leipälä and Nevalainen 1989; Shih et al. 1996; Duman and Or 2004).

*Corresponding author. Email: gxk@cs.nott.ac.uk

ISSN 0020-7721 print/ISSN 1464-5319 online 
When hundreds (possibly thousands) of electronic components of different shapes and sizes have to be placed at specific locations on a PCB, finding an optimal robot travelling route is a complex scheduling task ( $\mathrm{Su}$ and $\mathrm{Fu}$ 1998). Many researchers have modelled the component pick-and-place sequencing problem as a travelling salesman problem (TSP). Therefore, like the TSP, this problem is also NPHard and the majority of practical instances are difficult to solve to optimality in a reasonable time (De Souza and Lijun 1995; Ellis, Vittes and Kobza 2001). Indeed, the general PCB assembly problem is at least as complex as the TSP, which is known to be NP-complete (Nelson and Wille 1995).

Moyer and Gupta (1996b) argued that the PCB assembly problem is easy to describe, but due to the computational complexity of the sub-problems involved, in practical terms, it is hard to solve to optimality using mathematical programming approaches. Nelson and Wille (1995) stated that an exact solution using optimisation theory is unrealistic. For example, the component pick-and-place sequencing problem is a quadratic integer program that is difficult to solve using exact methods for even unrealistically small problems (Liggett 1981). The complexity of the problem is due to the interrelated sub-problems where the quality of the component pickand-place sequence is dependent upon the feeder setup and component retrieval sequence, and vice versa (Bard, Clayton and Feo 1994). Indeed, the concurrent movement of many machine parts (such as turret rotation, feeder carrier and $\mathrm{PCB}$ table) requires a full examination of all feasible combinations of feeder setups and component retrieval sequences in order to determine the best feeder setup and component retrieval sequence for each feasible solution of the component pick-and-place sequence. Moreover, the component pick-and-place sequencing problem is also tightly intertwined with the nozzle optimisation problem where seeking a good component pick-and-place sequence, without considering nozzle optimisation, might lead to unnecessary (possibly many) nozzle changes, which is very inefficient. In addition, there are many other issues that should be considered in optimising these sub-problems such as the grouping of components in a sub-tour (i.e. what components should be picked-and-placed together in each route if there is more than one pipette/nozzle per head); the speed differences among PCB table, feeder carrier and head movement; component transportation time; simultaneous pickup; etc.

De Souza and Lijun (1995) stated that exact methods are unsuitable for this problem and, as a consequence we have to consider heuristic and meta-heuristic approaches so that we can find good quality solutions in reasonable times. As an alternative, Moyer and Gupta (1996a) recommended simplifying the problem. For example, Ball and Magazine (1988), Gavish and Seidmann (1988), Leipälä and Nevalainen (1989), Chiu, Yih and Chang (1991), Ahmadi (1993), Van Laarhoven and Zijm (1993), Bard et al. (1994), Crama et al. (1996, 1997), and others have split the problems into a series of sub-problems in order to reduce the size of the search space.

Ayob and Kendall (2002a, 2008), carried out a survey of machine classifications and addressed optimisation issues based on the characteristics and operational methods of the SMD placement machines. The work related the machine characteristics and operational methods with the heuristics that have been applied. To complement these surveys, this article surveys a single machine optimisation problem that highlights optimisation issues in each sub-problem. The optimisation problems are classified into five subproblems, revealing some of the issues from each category, these being feeder setup, component placement sequencing, nozzle optimisation, component retrieval plan and motion control sub-problems. We aim to provide other researchers with a better understanding of the various optimisation issues in this field, and subsequently enable them to design and utilise heuristics, which are more appropriate to the realworld scheduling problem.

\section{The SMD placement machine}

The first pick-and-place SMD machines were introduced in the 1980s. These machines had only one placement head (Bentzen 2000). Many other types of machines are now available including sequential pick-and-place, rotary disk turret, concurrent pickand-place, dual-delivery, multi-station, multi-head, etc. (Grotzinger 1992; Khoo and Loh 2000; Gastel 2002; Ayob and Kendall 2008). As different SMD machines exhibit different characteristics, Wang, Nelson and Tripak (1999), Burke, Cowling and Keuthen (2001) and Ayob and Kendall (2008) argued that the PCB scheduling process is heavily influenced by the particular SMD machine being used.

Typically, each placement machine is fitted with feeder carrier(s) (or feeder magazine), PCB table(s), head(s), nozzle(s) (tool or gripper), pipette(s), tool magazine(s) (or tool bank), camera and trash bin. Figures 1 and 2 show one type of SMD placement machine (the picture was taken at the DIMA (DIMA SMT Systems, NL, B.V., Beukelsdijk, 5753 PA Deurne. (url: http://www.dimasmt.nl/)) factory, Holland). Depending on the machine specification, the feeder carrier, PCB table and head can either be fixed or moveable. 


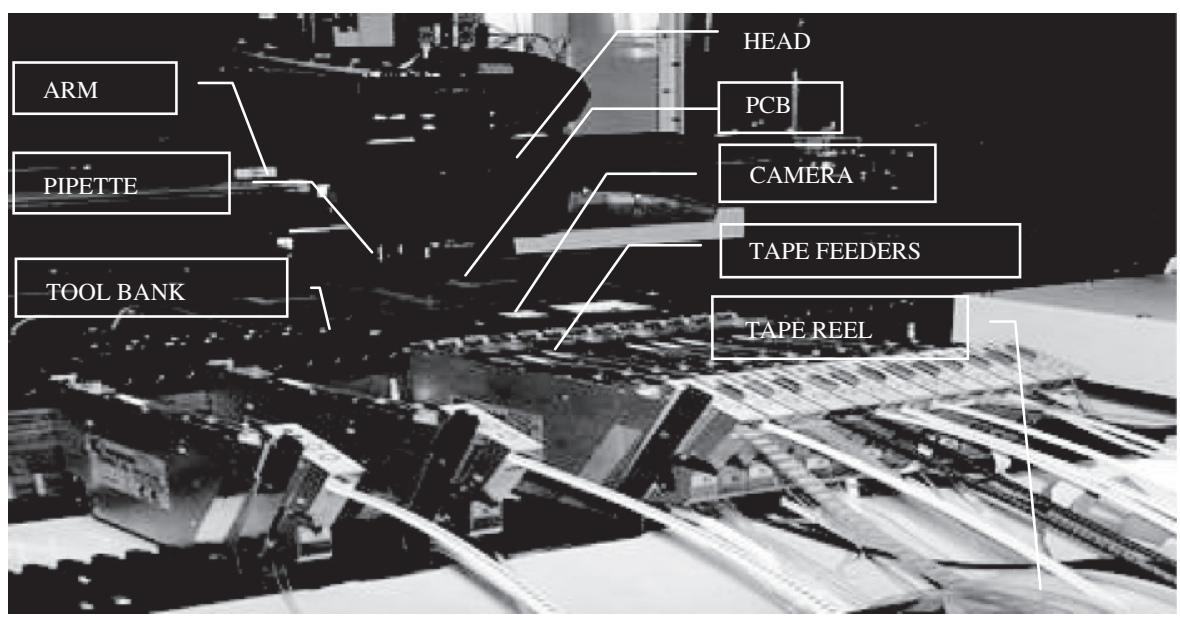

Figure 1. An example of an SMD placement machine (Dima HP-110).

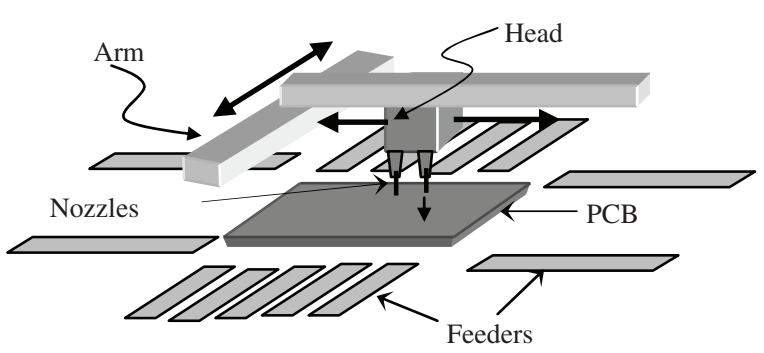

Figure 2. A multi-head SMD placement machine.

The feeder carrier is mounted on one, two, three or four sides of the machine and holds several feeder banks. The feeder bank has several feeder slots where the component feeders are located. The component feeders provide the machine with a continuous supply of components. The component feeders are arranged according to a predetermined arrangement. Figure 3 shows some of the available component feeders (pictured at the DIMA factory), which has various types of component packaging: tape, sticks and trays (or waffle). Tape reel feeders feed components that are packed in embossed, paper or surf tape. Depending on the component size, typical tape widths are 8, 12, 16, 24, 44, 56 and $72 \mathrm{~mm}$ (Bentzen 2000). Several slots may be occupied by one tape reel feeder (Sun, Lee and Kim 2005). If the components are supplied in sticks or tubes, then stick feeders are used to feed the components. Bentzen (2000) recommended avoiding using components with stick feeders for mass production, due to the delicate handling they require. The tape reel and stick feeders are arranged on the feeder slots of the feeder banks/carriers. Larger components, which are supplied in trays, are fed using tray feeders. Some machines allow a single tray to be placed into the machine feeding area whilst others use an automatic tray-handling unit. The use of a tray feeder further increases the optimisation problem that need to be addressed. A platform (that holds the trays component feeders) changeover takes about $10 \mathrm{~s}$ (for the HP-110 machine, for example). Therefore, optimising the tray feeder operation, if they are necessary, becomes a critical stage of the optimisation process.

The placement arm, that is equipped with head(s), is responsible for transporting components from feeders to PCB points. Located at the end of each head is a pipette(s), which hold a nozzle(s). The pipette(s) and nozzle(s) are used to grasp the components for the pick-and-place operations and moves in the $Z$ direction (up-down). Each head may have more than one pipette and each machine may have more than one head. There are various types of placement heads, such as a rotating turret head, or a positioning arm head (Wang et al. 1999; Ayob and Kendall 2002a, 2008).

Each component packaging type can be associated with more than one nozzle type and vice-versa. The problem is more complicated as one component type can have more than one type of packaging. This means that each PCB point on the board can receive only one component type, but those components may have different packaging. The component packaging can be recognised and aligned without a vision camera (i.e. using mechanical alignment on the fly), using a small vision camera and/or a large vision camera, depending on the component packaging specification. When a defective component is detected, the machine head will discard it into the trash bin.

Different nozzle sizes are required for the various types of component packaging. An automatic nozzle change system is used to ensure that the correct nozzle 


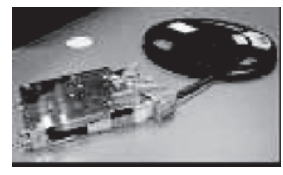

(a) Tape reel feeder

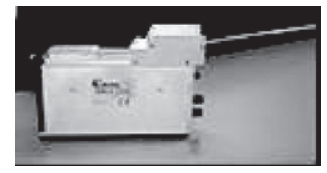

(b) Stick feeder

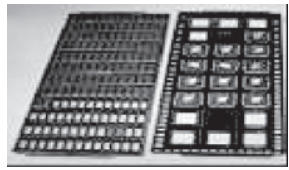

(c) Tray feeder

Figure 3. Example of component feeders.

is used for the relevant component. The tool bank, located on the machine, provides the ability to carry out a nozzle change, as part of the machine's normal operation. Some nozzles, which usually use a vacuum, are used to transport components from the component feeders, whereas special nozzles with a mechanical alignment capability are necessary for the handling of odd-shape components (Bentzen 2000).

The PCB table(s) is required to position the $\mathrm{PCB}(\mathrm{s})$ during the placement operation. The table(s) could be stationary, a conveyor system, or an $\mathrm{X}-\mathrm{Y}$ motion table.

\section{Production planning problem in PCB assembly}

As discussed in Crama, Klundert and Spieksma (2002), production planning problems can be categorised into eight sub-problems. These are:

(1) Assigning PCB types to product families and to machine groups.

(2) Allocating component feeders to machines.

(3) Partitioning component locations on the PCB to indicate which components are going to be placed by each machine (for each PCB type).

(4) Sequencing the PCB types.

(5) Assigning component feeders to slots on the feeder carrier (feeder setup).

(6) Sequencing the component pick-and-place operations.

(7) Component retrieval plans.

(8) Motion control specification.

These sub-problems are tightly intertwined. Hence, a dilemma exists as to which one should be solved first. Some researchers solved the problem in an iterative manner, whilst others use a hierarchical problem solving approach, integrated approach or just solve a single (or few) sub-problem(s) by assuming that the others have been determined. Moyer and Gupta (1997) and Crama et al. (2002) argued that technological differences among various type of SMD placement machines can influence the nature of some of the problems to be solved and the formulation of the associated models. Therefore, it is difficult to determine a general formulation for each sub-problem. For example, the component pick-and-place time of turret-type SMD placement machines (see, e.g. Moyer and Gupta 1996a, b, 1997; Ellis et al. 2001; Ho and Ji 2003) is determined by the maximum time taken between the turret rotational time (a type of placement head), PCB travelling time and feeder carrier travelling time. Whereas, for the multi-head placement machines that has a stationary feeder carrier, a fixed PCB table and a positioning arm head that is equipped with few pipettes (Altinkemer, Kazaz, Koksalan and Moskowitz 2000; Jeevan et al. 2002), the component pick-and-place time is determined by the many other factors such as the grouping of PCB points (also referred to as placement points) to a sub-tour, nozzle assignment, gang-pickups and simultaneous vision. Other types of SMD placement machines are dualdelivery, multi-station and sequential pick-and-place (Ayob and Kendall 2002a, 2008).

Crama et al. (2002) also addressed the problem of having insufficient problem descriptions in the literature. They recommended that authors should mention (at least) the following key elements in their papers:

(1) Shop layout (decoupled workcells, one or several assembly lines, etc.).

(2) Characteristics of the product mix (high volume-low variety, low volume-high variety, etc.).

(3) Setup policy if more than one board type is to be produced.

(4) Relevant characteristics of the SMD placement machines (sequential, concurrent, etc.).

(5) Decision to be taken, according to the eight sub-problems.

Other surveys have been conducted by McGinnis et al. (1992), Ahmadi (1993), Ji and Wan (2001). Ahmadi (1993) devised a hierarchy of decision problems and developed a model to optimise the decision-making process in PCB manufacturing. Ji and Wan (2001) and McGinnis et al. (1992) categorised the production planning problems into three stages being grouping (i.e. assigning PCB types to product families and to machine groups); allocation (i.e. identifying which machine in the assembly line to assemble which components) and arrangement and sequencing (i.e. assigning component feeders to slots on the feeder banks and sequencing the component's pick-and-place operations). 


\section{Single machine optimisation}

In this work, we focus on the problem of a single machine with a single board type. In the context of a hierarchical decomposition approach, Magyar et al. (1999) argued that the single machine optimisation problem is considered as the lowest operational level.

Crama et al. (2002) classified the single machine problem into four sub-problems, these being feeder setup, component pick-and-place sequencing, component retrieval plan and motion control-whereas, in this work, we include one more sub problem, that is nozzle optimisation (i.e. five sub-problems in total). On the other hand, Magyar et al. (1999) encountered four sub-problems in the case of single machine optimisation, these being: feeder setup, component pick-andplace sequencing, component retrieval plan and nozzle optimisation. Some works have addressed the problems of feeder setup and placement sequence independently by making assumptions about the rest of the problem, and some prefer to solve both problems as an integrated solution (Ellis et al. 2001). A hierarchical problem solving approach has also been studied (Magyar et al. 1999).

Figure 4 illustrates the operational relationship between the five intertwined sub-problems. For example, the sequence of nozzle changes (nozzle optimisation procedure), will affect the arrangement of component feeders on the feeder carrier and the sequence of component pick-and-place and viceversa. That is, when the sequence of nozzle changes have been determined, the arrangement of component feeders on the feeder carrier should consider the sequence of nozzle changes in order to have a good feeder arrangement which can minimise the component pickup operation. This can be done by, for example, maximising simultaneous pickups, minimising pickup distance, etc. Similarly, when the sequence of nozzle changes have been determined, the sequence of component pick-and-place operations is dependent on the sequence of nozzle changes, due to component specific nozzles (i.e. a component type can only be picked-and-placed by a set of nozzles).

\subsection{Motion control}

When considering an SMD placement machine that has a moveable head, a feeder carrier and a PCB table, one should consider where the effective pick-and-place points are. That is where the robot arm meets the feeder carrier (or the PCB) to pick (or place) a component. The robot (that is the arm and head) is able to move in both $\mathrm{X}$ and $\mathrm{Y}$ directions concurrently in order to pick-and-place a component. The feeder carrier and the PCB table are moveable in the $X$-axis to position the component pickup coordinate and the placement coordinate of the $\mathrm{PCB}$, respectively. The robot, PCB table and feeder carrier can move concurrently. The robot travels between the feeder carrier(s) and the PCB table for picking and placing a component, respectively.

Until now, there have not been many publications, which have reported improving motion control. This might be due to the fact that many SMD placement machines use fixed pick-and-place points since not many of them have moveable heads $(\mathrm{X}-\mathrm{Y})$, feeder carriers and PCB tables. For example, a turret type SMD placement machine has a rotating turret that rotates from a fixed pickup location to the fixed placement location.

Some works that have focused on motion control are Su, Wang, Egbelu and Cannon (1995), Wang, Ho, $\mathrm{Fu}$ and $\mathrm{Su}$ (1995, 1997), Wang (1996), Wang, Ho and Cannon (1998), Bonert, Shu and Benhabib (2000), $\mathrm{Fu}$ and $\mathrm{Su}$ (2000), Hop and Tabucanon (2001a, b) and Ayob and Kendall (2005). These publications suggest a dynamic pick-and-place (DPP) point to avoid robot waiting time. The approach allows the robot to pick-and-place a component at any location rather than fixed pickup-and-placement (FPP) locations. Most of these works solved the problem for sequential pick-and-place machine except Bonert et al.

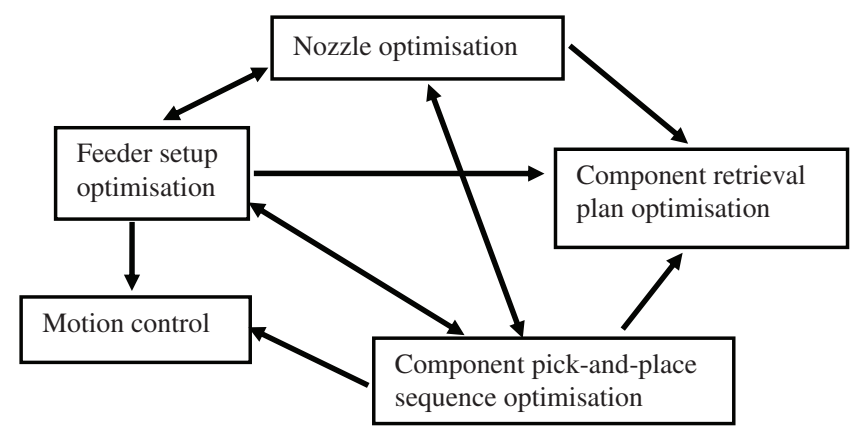

Figure 4. The relationship of the five sub problems. 
(2000), which dealt with a dual-delivery placement machine.

\subsection{Nozzle optimisation}

Nozzle optimisation, in the context of single SMD placement machine optimisation, involves searching for an effective nozzle assignment and sequencing/ switching by minimising the number of nozzle changes in order to improve machine throughput. In minimising the dual-delivery placement machine, Tirpak et al. (2000) defined a nozzle minimisation problem as an assignment of a nozzle based on the weights associated with each nozzle type. This involves finding the best distribution of the nozzle(s) on the heads, which yields the minimum pickup time.

When the SMD placement machine has more than one pipette/nozzle per head (or even a single nozzle per head), choosing an effective nozzle group (or a nozzle) is important in order to improve the pick-and-place operations and to minimise the number of nozzle change operations. Having a proper nozzle group assignment might lead to more simultaneous pickup operations, minimise feeder carrier movement, as well as robot arm and/or PCB table movements. This can ultimately improve the machine throughput. A nozzle changeover operation is very time consuming (Crama, Kolen, Oerlemans and Spieksma 1990; Safai 1996; Shih et al. 1996; Lee, Lee, and Park 1999; Magyar et al. 1999; Jeevan et al. 2002). For example, the HP-110 (Dima SMT Systems 2003) takes about $2 \mathrm{~s}$ for a nozzle changeover operation.

According to Magyar et al. (1999), optimising the pick-and-place operation without considering the nozzle switching operations may not be efficient, since it may cause many unnecessary nozzle changes that will significantly reduce the machine throughput. The problems of minimising nozzle switching and minimising the pick-and-place operations are tightly intertwined and should not be solved independently. Nevertheless, Sun et al. (2005) argued that the nozzle changeover operation is not directly affected by the component allocation and feeder setup decision. As far as we are aware, none of the research (in the context of a single SMD placement machine minimisation) has tackled the nozzle minimisation problem individually. Some works that addressed the importance of nozzle minimisation are Chang and Terwilliger (1987), Ahmadi, Grotzinger and Johnson (1988, 1991), Crama et al. (1990), Safai (1996), Shih et al. (1996), Magyar et al. (1999), Tirpak et al. (2000) and Jeevan et al. (2002). They solved the nozzle minimisation problem, together with the problem of sequencing the pick-and-place operation and/or feeder setup. To date, there has been relatively little research that has addressed the minimisation of nozzle switching. Even an exhaustive survey by Crama et al. (2002) did not address this problem. A survey by McGinnis et al. (1992) also found that only limited research employ component specific nozzles.

Some researchers, for example, Bard (1988) and Crama et al. (1990), Crama, Kolen, Oerlemans and Spieksma (1994), considered minimising nozzle switches in the context of flexible manufacturing. A crucial problem of nozzle management (minimising) in a flexible manufacturing environment is to identify the sequence of parts to be produced, and which nozzles to allocate to the machines so as to minimise the number of nozzle setups across a line of several machines (Crama et al. 1994). For example, Crama et al. (1990) proposed a heuristic hierarchical approach to the problem of minimising the throughput rate of a line of several SMD placement machines by first assigning the nozzle to the machines, and then performing the component allocations.

In solving the component placement sequence problem, Chang and Terwilliger (1987) applied a rule-based approach, where one of the rules aimed to minimise the nozzle changes. Unfortunately, they did not present any results.

When eliminating the head contention (i.e. the case when more than one head requires the same nozzle at the same time), Safai (1996) indirectly reduced the nozzle changes. They represented the cost of nozzle changes in terms of placement cost and included the cost in the objective function, which aims to minimise the assembly cycle time. The assembly cycle time is a total time taken by the machine to assemble all the components on a PCB.

Shih et al. (1996) employed an expert system approach to minimise a multi-station SMD placement machine by first minimising the nozzle changes, then minimising the component pick-and-place sequence. In order to minimise the nozzle changes, they group the components in a placement sequence so that the components using the same nozzle type can be placed consecutively. They designed five rule sets for nozzle minimisation. These are:

(1) Rule set 1: Grouping the placement steps based on the station where the placement steps will be performed. Next, the nozzle minimisation procedure can begin with respect to the individual station.

(2) Rule set 2: Sequencing the nozzle sets used based on their handling capabilities such that more clearance is provided in placing large components while simultaneously minimising the frequency of nozzle changes. The size of 
the nozzle affects the clearance required at a placement location on the PCB.

(3) Rule set 3: Arranging the nozzle changes in ascending order of component mass.

(4) Rule set 4: Ensuring the placing of unleaded components (components without legs) prior to leaded components.

(5) Rule set 5: Sequencing the component placement in ascending order of component mass.

By using the output from the nozzle minimisation stage, Shih et al. (1996) minimised the component pickand-place operation using a simple descent search. Their results were verified by machine experts and are very promising, which might contribute to about 15 working days of time saving over a year.

A hierarchical problem solving approach has been applied by Magyar et al. (1999) to solve the problem of determining the sequence of component pickup-andplacement; and schedule the assignment of different nozzles to the pipettes. They first create nozzle layers to indicate the assignment of nozzles to pipettes for each sub-tour. In searching for a good nozzle layer, they used a weighted cost function to overcome the trade-off issues between minimising the nozzle changes and minimising the number of sub-tours. They argued that nozzle changes are costly. Likewise additional sub-tours increase the camera costs. Reducing nozzle changes will increase the number of placement groups and vice-versa. Their algorithm iteratively selects the sequence of nozzle changes (which starts with the minimum number of nozzle changes) and then increases the number of nozzle change operations and determines the number of sub-tours in order to reduce the assembly cycle time. Results tested on real industrial problems show that their approach achieved savings of assembly cycle times by $7.5 \%$.

By listing the type of components to be assembled and the associated nozzles used, Tirpak et al. (2000) assigned the nozzles to the heads by considering the best distribution of the nozzles to the head. They improve the initial nozzle setups by randomly selecting two nozzles of different sizes, and swapping their positions on the revolver head (each revolver head has 16 nozzles).

Jeevan et al. (2002) used a genetic algorithm (GA) to minimise the component pick-and-place sequence of the multi-head placement machine by considering the importance of minimising the tool change operation. They represent a distance of a TSP tour (i.e. a total pickup-and-placement distance) as a fitness function. In order to eliminate any unnecessary nozzle changes, they use all the components that can be placed by a certain nozzle before changing the nozzle. However, since a component type (or package) can be picked up by more than one nozzle type, and the tool changing time was excluded from the fitness function evaluation, the aim of reducing tool changes might not be achieved.

In conclusion, other researchers might consider including the cost of nozzle changes in the objective function when minimising assembly cycle times.

\subsection{Component pick-and-place sequence optimisation}

Suppose that the feeder setups, the component retrieval plan, the motion control and the nozzle sequencing have been determined. In this case, we need to search for a good component pick-and-place sequence in order to maximise the machine throughput. Many papers (Leu et al. 1993; Jeevan et al. 2002) define component pick-and-place optimisation as finding a shortest route to pick-and-place the electronic components onto the PCB. This is only true if other factors such as nozzle changes, feeder transportation time (i.e. time taken by the feeder to transport the component to the pickup position), gang pickups (i.e. simultaneous pickup), tray feeder changes (i.e. platform changes), etc. are ignored. Therefore, it is more precise to define component pick-and-place optimisation as finding a shortest time to pick-andplace the electronic components onto the PCB (Ng 1998).

Due to technological advancements, the component density on a PCB has gradually increased. That is, the distance between the PCB points has decreased. As a result, Sun et al. (2005) argued that the decision of feeder setups and pickup sequences are more crucial in determining the efficiency of the machine compared to the component placement sequencing. However, it may be the case that minimising nozzle changes and component placement sequencing are also crucial. Based on our discussions with PCB assembly companies, it is desirable not to change the feeder setup unless it is unavoidable. Therefore, in this case, the optimisation of the component pickup-and-placement sequencing plays a significant factor in improving the throughput of SMD placement machines. However, as the speed of the robot arm (i.e. the arm and head) of the latest machines is very fast and the component density on PCBs has increased, minimising the robot travelling distance is becoming a less significant factor for improving machine throughput. Indeed, due the acceleration/deceleration of the robot arm, the time taken for the arm to move short or longer distances might be fairly equal. Therefore, it is ineffective to just minimise the robot travelling distance in order to improve the machine throughput. For the purpose of 
optimising the component pick-and-place operation, exact information about the machine speed, acceleration/deceleration rate, etc. is not necessary (as the machine is embedded with a control software for accurate movements/operations). The average machine operation time is adequate in guiding the search for a better quality schedule (Ayob and Kendall 2004). Moreover, if the machine speed, acceleration/deceleration rate, etc. is included, it might necessitate a more complex formulation of the objective function.

Many researchers have modelled the component pick-and-place sequencing problem as a TSP (Drezner and Nof 1984; Gavish and Seidmann 1988; Chan and Mercier 1989; Leipälä and Nevalainen 1989; McGinnis et al. 1992; Chan 1993; Bard et al. 1994; De Souza and Lijun 1994, 1995; Francis, Hamacher, Lee and Yeralan 1994; Khoo and Ng 1998; Tirpak et al. 2000; Jeevan et al. 2002; Kumar and Luo 2003; Duman and Or 2004); whilst Ball and Magazine (1988) treated it as a rural postman problem. Several researchers (Altinkemer et al. 2000; Grunow, Günther, Schleusener and Yilmaz 2004) viewed the problem as a vehicle-routing problem and Leipälä and Nevalainen (1989) treated it as a three-dimensional asymmetric TSP. Nevertheless, many researchers (Shih et al. 1996; Yeo, Low and Yong 1996; Magyar et al. 1999; Ho and Ji 2003, 2004, 2006; Sun et al. 2005) solved the pickand-place sequencing problem as a unique problem since the problem relies heavily on the machine characteristics.

The optimisation of a TSP aims to find a route by visiting each city exactly once while minimising the total distance travelled. By defining chip locations ( $\mathrm{PCB}$ points) as cities and the time between chip insertions (or placements) as distances, the component pickup-and-placement sequencing problem can be formulated as a TSP (Chan and Mercier 1989). In reality, it is not a straightforward mapping since the effectiveness of the component pick-and-place sequence is not only dependent on the robot (and/or PCB table, feeder carrier) travelling distance. Good quality schedules may be obtained by modelling the problem as a TSP but but it may be possible to obtain much better solutions if the other crucial factors such as nozzle changes, feeder transportation time, gang/ simultaneous pickups etc. are also considered. However, this poses a much greater optimisation challenge, which might not be solved by just modelling the problem as a TSP. In principle, crucial optimisation factors are machine dependent. Modelling the pick-and-place operations of certain machine types as a TSP might be suitable but this might not be applicable to other machine types. However, to be more realistic, we would recommend considering some crucial optimisation factors such as robot travelling distance, nozzle changes, feeder transportation time, simultaneous pickup, etc. for calculating the time between chip placements (which represent distances for TSP).

A rural postman problem, which is a generalisation of the Chinese postman problem, is a problem of finding an optimum (or least-cost) postman tour covering all the edges (streets) in the network, in which the underlying street network may not form a connected graph (Pearn and Wu 1995; Kang and Han 1998). We may view a set of pickup-andplacements points of each nozzle as a connected tour but which are isolated from the tour of other nozzles. Thus, we can include the time of nozzle changes when changing the tour. Again, we would recommend minimising the time needed to perform the pick-andplace operations (which may include component recognition, gang pickup etc.) instead of just minimising robot travelling distance.

A vehicle-routing problem is the problem of determining a set of routes for vehicles which depart from, and return to, a single depot, for a fleet of vehicles (Van Breedam 2001).

Ball and Magazine (1988) was the first work, which attempted to solve the component pick-and-place problem for a moving head, stationary PCB table and feeder carrier. Thereafter, many studies of optimising the component pick-and-place problem have been reported. For example, Mettalla and Egeblu (1989) employed a rule-based approach for solving the component pick-and-place problem. The rules were based on dominance properties of robot arm movement, feeder carrier movement etc.

Sanchez and Priest (1991) addressed four basic insertion/placement rules:

(1) To avoid interference, smaller size components should be placed prior to larger size components.

(2) All the same type of components are assembled in one pass.

(3) Assemble components of identical sizes and shapes, and then assemble the other components of non-similar size and shape.

(4) Choose a near-optimal sequence to minimise the PCB table movement.

In other work, Wong and Leu (1993) also addressed four basic component pick-and-place and feeder setup rules that are usually adopted:

(1) Sequence the component placement for minimum routing time.

(2) Arrange feeder reels so as to minimise component pickup time.

(3) Place identical SMDs in one pass. 
(4) Sequence placement according to component size.

However based on discussions with a machine manufacturer (DIMA, http://www.dimasmt.com) one should avoid a consecutive pick up of the same component type from the same feeder slot since this incurs an extra picking cost due to a component feeder transportation cost (i.e. the next component has to be moved into place), which is usually more than the cost of moving to pickup from another feeder slot. Therefore we would suggest that the pick-and-place sequence should be arranged such that the components that use the same nozzle type(s) are assembled consecutively instead of assembling the same type of components or identical SMDs in one pass. This strategy might also help to reduce nozzle change operations.

Leu et al. (1993) associated the planning problem (particularly the component pick-and-place sequencing problem) with the characteristics of various SMD placement machines. They identified three planning problems as shown in Table 1. The third column in Table 1 shows the relationship between the machine characteristics (Leu et al. 1993) and the classification of the SMD placement machine as in Ayob and Kendall (2002a, 2008). However, due to a technology change, the SMD placement machine characterised for the TSP (refer to Table 1) is not classified in Ayob and Kendall (2002a). This might be an old machine and as far as we concerned, none of the work focuses on this machine type except Leu et al. (1993). The planning problem of the first machine (refer to Table 1) was treated as a TSP since the issue is to find a sequence of placement head(s) in visiting all PCB point locations such that the travelling time is minimised, regardless of the pickup operations. Whereas, due to the fact that the cost of component placement sequencing is very dependent on the feeder setup, the planning problem of the second machine (refer to Table 1) was modelled as a pick-and-place problem. Subsequently, the planning problem of the third machine (refer to Table 1) was modelled as a Moving Board with Time Delay Problem because there is often a time delay caused by either the feeder carrier movement or the turret rotation when the PCB travels between two consecutive placement points and therefore the problem cannot be treated as a simple TSP.

By considering a case where certain placement sequences are not acceptable (which may cause a placement head damaging the previously placed components during a placement operation), Duman and Or (2004) treated the component pick-and-place sequencing problem as a precedence constrained travelling salesman problem (PCTSP). They first formulated the problem as a pure chebyshev (i.e. $\max (|\Delta x|,|\Delta y|)$, where $|\Delta x|$ and $|\Delta y|$ are the distances between two points in X-coordinate and Y-coordinate, respectively) TSP using Convex-Hull and Or-opt algorithms (Duman and Or 2004). Then, a damage reduction procedure is designed to eliminate damage to components in the resulting TSP tour. They argued that the approach is successful in eliminating component damage and is able to facilitate full automation of the operations.

Some approaches such as tabu search ( $\mathrm{Su}, \mathrm{Hu}$ and Fu 1998; Csaszar, Tirpak and Nelson 2000b), GAs (Leu et al. 1993; Khoo and Ng 1998; Khoo and Ong 1998, Ong and Tan 2002; Chyu and Chang 2008), neurofuzzy modelling (Tsai, Yang and Hou 2005), expert system (Huang and Srihari 1994), knowledgebased (De Souza and Lijun 1994), rule-based (Mettalla and Egeblu 1989) and neural networks (Su and Srihari 1996) are among the effective approaches when optimising component pick-and-place sequence and feeder setup.

\subsection{Feeder setup optimisation}

The question of where (i.e. in which slots) the feeder reels (and/or sticks) should be attached on the feeder carrier of each placement machine is referred to as feeder setup (Tirpak et al. 2000), the feeder rack assignment problem (Klomp, Klundert, Spieksma and Voogt 2000), the component-feeder arrangement (Khoo and Loh 2000), the reel positioning problem (Ahmadi, Ahmadi, Matsuo and Tirupati 1995; Ohno,

Table 1. The relationship among SMD placement machine characteristics, planning problem types and machine classification.

\begin{tabular}{|c|c|c|c|}
\hline & Problem type & SMD placement machine characteristics & Machine type \\
\hline 1 & Travelling salesman problem (TSP). & $\begin{array}{l}\text { Stationary head, } \mathrm{X}-\mathrm{Y} \text { table, direct feeding of } \\
\text { components to assembly head. }\end{array}$ & Unclassified. \\
\hline 2 & Pick-and-place problem. & $\begin{array}{l}\text { Moving head, stationary table, stationary } \\
\text { feeders. }\end{array}$ & Sequential pick-and-place. \\
\hline 3 & Moving board with time delay problem. & $\begin{array}{l}\text { X-Y table, moving feeders, supply of compo- } \\
\text { nents with a multi-head turret or a moving } \\
\text { head between two fixed locations. }\end{array}$ & Turret-type. \\
\hline
\end{tabular}


Jin and Elmaghraby 1999), feeder assignment (Loh, Bukkapatnam, Medeiros and Kwon 2001; Hop and Tabucannon 2001b; Li, Hu and Tian 2008), feeder allocation (Altinkemer et al. 2000), magazine assignment (Ahmadi et al. 1988) or feeder configuration (Duman and Or 2007). In this article we use the term feeder setup to refer to this problem. The feeder setup decision determines where the component feeders are located on the feeder slots of the feeder bank/feeder carrier. As the larger size components are usually fed using tray feeders, which are only available when the appropriate tray is placed into the machine feeding area, it is recommended that the inclusion of a tray feeder arrangement into the feeder setup problem (especially if this involves many platforms) would be beneficial.

Sun et al. (2005) argued that unlike the TSP, the evaluation of the solution quality of the feeder setup is not straightforward. For example, according to Ball and Magazine (1988), the cost of a particular feeder setup depends on the sequence of pick-and-place operations. That is, we need other interrelated decisions such as a decision for nozzle assignment and sequencing, component pickup-and-placement sequencing, gantry scheduling etc. Nevertheless, Sun et al. (2005) said that the other decisions should be solved in order to avoid disturbances and maintain the consistency of the evaluation, while searching for an improved feeder setup. Similarly, when optimising component pick-and-place sequencing, other optimisation problems such as a feeder setup should be solved in a simple manner or assume they have already been solved. For example, Leipälä and Nevalainen (1989), Moyer and Gupta (1996a) and Dikos, Nelson, Tirpak and Wang (1997) solved the feeder setup problem based on the assumption that the placement sequence was predetermined or fixed.

When the placement sequence is fixed, the feeder setup can be formulated as a quadratic assignment problem (Leipälä and Nevalainen 1989). Francis, McGinnis and White (1992) and Moyer and Gupta (1996a, b) have modelled the feeder setup problem of a turret-type machine in this way, since the feeders are assigned to slots on the feeder carriage and the cost of the assignment is affected by the location of other feeders. Whereas, Drezner and Nof (1984) and Foulds and Hamacher (1993) modelled the feeder setup problem as a bin location assignment that was formulated as a single-facility location problem.

Many researchers have attempted to enhance the feeder setup including Leipälä and Nevalainen (1989), Crama et al. (1990), Grotzinger (1992), Ji, Leu and Wong (1992), Foulds and Hamacher (1993), Leu et al. (1993), Sadiq, Landers and Don Taylor (1993), DeSouza and Lijun (1994), Ji, Wong, Loh and Lee
(1994), Moyer and Gupta (1996a, b), Sohn and Park (1996), Yeo et al. (1996), Dikos et al. (1997) and Sun et al. (2005). Most researchers highlight that the crucial moves, which affect the optimisation, are the feeder carrier movements (Grotzinger 1992; Ahmadi and Mamer 1999; Kumar and Luo 2003), which is the case for moveable feeder carriers. Therefore, optimising the feeder setup, which can lead to the optimisation of the feeder carrier movement, is also a crucial factor when optimising machine throughput.

Sohn and Park (1996) simultaneously solved the component pick-and-place sequence and feeder setup of the turret-type SMD placement machine. They assigned component feeders to slots based on the frequency of use, and then use the obtained feeder setup to solve pick-and-place sequences.

Based on the predetermined component placement sequence, Moyer and Gupta (1996a) solved the feeder setup of turret-type placement machines by assigning component feeders to slots based on the switching between component types. Next, they improve the solution by exchanging pairs of slots. They aim to minimise the feeder travelling distance. Their feeder setup was superior to Leu et al. (1993) in terms of feeder travelling distance. There is an argument as to whether these approaches will lead to a reduction in the assembly cycle time. Reducing the feeder travelling distance can only reduce the time required for feeders to supply the required components to the turret head. Of course, this will help minimise the assembly cycle time if the feeder movement time is the dominating factor. Unfortunately, Gastel (2002) revealed that this is not the case since the PCB X-Y movement is also a determining factor (in most cases) of the throughput rate of turret-type placement machine compared to the turret rotation time. Since the movement of the feeder carrier and PCB table of the turret-type machine happen simultaneously, it may be worthwhile minimising the assembly cycle time instead of minimising the feeder travelling distance. Therefore, the feeder setup is dependent on the component pick-and-place sequencing. Nevertheless, if the feeder setup is designed for multiple PCB types, the aim of minimising the feeder travelling distance for improving the machine throughput is appropriate.

In other work, Moyer and Gupta (1996b) proposed the acyclic assembly time (AAT) algorithm to concurrently improve the quality of the component pickand-place sequence and feeder setup. In the case where the PCB is still moving to locate the proper placement point, the AAT model allowed the other mechanism to advance to the next position in order to avoid machine idling. Again, Moyer and Gupta (1996b) argued that on average, their approach is superior to Leu et al. (1993) and De Souza and Lijun (1994). 
Yeo et al. (1996) used a rule-based approach to concurrently solve the component placement sequencing and feeder setup problem of the turret-type machine. To optimise this machine, Yeo et al. (1996) argued that the next pickup operation should be at most one slot distance away. Therefore, based on the component placement sequence, they arranged the components on the feeder slots by trying to maximise consecutive pickups at adjacent feeder slots. Their objective is to reduce the $\mathrm{X}-\mathrm{Y}$ PCB table and feeder carrier movements in order to maximise the machine throughput. They allow feeder duplication (that is, the same component type can exist at various feeder locations), which could increase the chances of having more consecutive pickups at adjacent feeder slots. Solution qualities are measured using the total distance traversed by the $\mathrm{X}-\mathrm{Y}$ PCB table and the actual machine cycle time per PCB. Computational results showed that the proposed approach outperformed the Fuji machine optimisation software in terms of the PCB table movement and the actual machine cycle time. Based on a typical production of 1000 PCBs per day, the approach could yield an improvement of about 7000 PCBs per month.

Klomp et al. (2000) represent a feeder (and its corresponding cluster i.e. set of locations served by a single feeder) as a node in a complete graph. For each pair of nodes (feeders), say $j$ and $k$, they compute the length of edge $\{j, k\}$ as a Hamiltonian path. The length is an approximation of the processing time when component feeder $j$ and $k$ are assigned to adjacent slots on the feeder carrier. Klomp et al. (2000) solve the feeder setup problem by using an insertion heuristic and a basic descent two-opt local search. They claim that their approach is capable of generating good quality solutions, which are relatively close to the lower bound (about 20\% in the three machine case). This implies that much of the PCB table and feeder carrier movements fall within the turret rotation time. Since the turret rotation is an unavoidable movement, Leu et al. (1993) argued that the lower bound of the turrettype machine is achieved if the assembly cycle time is only dictated by the turret rotation time (i.e. one stepwise rotation time). However, to date, as far as we know, the question of how to calculate the lower bound of other machine types (i.e. dual-delivery, multistation, multi-head and sequential pick-and-place) is still an open question.

Khoo and Loh (2000) employed a GA to generate the component placement sequence and feeder setup by formulating it as a multi-objective optimisation problem. The prototype system demonstrated the ability to generate a component pick-and-place sequence and feeder setup which was slightly better than Leu et al. (1993).
Chyu and Chang (2008) used a spanning tree technique to investigate the use of feeder duplication. They concluded that the overuse of feeder duplication has a negative effect on the overall cycle time.

Ellis et al. (2001) proposed a constructive heuristic that groups together components with similar PCB table speed and turret rotation speed. They solve feeder setup and component placement sequencing problems for turret-type machines. They employed a surrogate function, which provided a method to approximate penalties for feeder carriage movements, changes in turret rotation speed and changes in PCB table speed. A two-opt heuristic is used to search for placement time improvements. Results indicate that the solutions are close to the lower bound and the computational time required to generate the initial solutions is minimal (less than 3 minutes).

Ho and Ji (2003 and 2004) used a GA approach (called HGA, i.e. Hybrid Genetic Algorithm) to represent a chromosome as two-link structures. Both links represent the sequence of component pick-andplace and feeder setup, respectively. They constructed initial solutions (i.e. initial chromosomes) for the first link using a nearest neighbour heuristic, whilst the second link is randomly generated. The first link is improved using an iterated swap procedure (Ho and $\mathrm{Ji}$ 2003, 2004), whilst a two-opt local search heuristic is applied to the second link. Roulette wheel selection is used to select chromosomes to undergo genetic operations. Their HGA also used a modified order crossover operator and two mutation operators (i.e. heuristic mutation and inversion mutation). They represent the total assembly cycle time as the fitness function. Ho and $\mathrm{Ji}$ (2003) argued that the HGA is superior to a simple GA used by Leu et al. (1993) with better initial solutions and final solutions (i.e. smaller population sizes and fewer iterations compared to Leu et al. (1993)).

By adopting a GA approach, Sun et al. (2005) successfully assigned component feeders to slots by maximising simultaneous pickups in order to minimise the number of pickups and, as a consequence, improved machine efficiency. The algorithm evenly allocated the component feeders to the two feeder carriers. They observed that empty slots between feeders help maximise simultaneous pickups; the feeders are close to each other so as to minimise the pickup travelling time; and the feeders are located close to the centre position of each feeder carrier such that the robot travelling time between the feeder carrier and the PCB point is minimised.

Grunow et al. (2004) arranged component feeders to slots based on the strength of the neighbourhood relations. Next, they improved the feeder setup using 
a random descent two-opt swapping procedure. Their results showed that the proposed heuristics are very efficient and can produce a high quality solution which is close to the theoretical lower bound. For example, for smaller size PCB's, they obtained an average deviation from the lower bound of $2.32 \%$.

DIMA (http://www.dimasmt.com/) have told the authors that scheduling tray feeders also poses a challenging optimisation problem. Components that are supplied in trays are only available when the appropriate tray is placed into the machine feeding area. Since a platform (that holds the tray feeders) change is very time consuming, optimising the tray feeder operation (if there are many platforms) is an important consideration. Without properly scheduling the component pickup operations from the tray feeders, it may cause machine idling time (i.e. the robot arm may frequently wait for the proper tray to be reloaded into the machine feeding area, which might take, for example, about $10 \mathrm{~s}$ for each tray changeover). This is very inefficient. As far as we are aware, this is an unexplored research problem. Many researchers ignore the tray feeder problem by assuming that there is no tray feeder in the machine or the components on the tray are always available in the machine, and treat them as a normal feeder. Based on our observation, most works on improving feeder setup focus on turret-type machine. This might indicate that, for turret-type machines, a feeder setup decision is more important compared to other machine types.

\subsection{Component retrieval plan optimisation}

If the feeder carrier slots hold several component feeders of the same type (feeder duplication), a decision has to be made as to which feeder slot the component type should be retrieved from by assuming that a feeder setup and a component pick-and-place sequence have been determined (Bard et al. 1994; Crama et al. 1996). This is a component retrieval problem. Crama et al. (2002) argued that the decision is heavily dependent on the modus operandi of the SMD placement machine. Bard et al. (1994) found a strong relationship between the feeder setup and the component retrieval problem.

Feeder duplication is a common practice that can substantially improve the machine throughput (Crama et al. 1996, 1997; Chen and Chyu 2003). Some other works that consider feeder duplication are Ahmadi et al. (1988), Bard et al. (1994), Francis et al. (1994), Klincewicz and Rajan (1994), Ong and Khoo (1999), DePuy, Ammons and McGinnis (2000) and Kazaz and Altinkemer (2003).

Bard et al. (1994) employed a forward dynamic programming approach to solve the problem.
The search for an optimal component retrieval plan was carried out using a branch and bound algorithm. However, by using an example and mathematical formulation, Crama et al. (1996) argued that the forward dynamic programming approach proposed by Bard et al. (1994) cannot possibly lead to a correct algorithm for component retrieval problem. Consequently, Crama et al. (1996) introduced a twophase polynomial-time dynamic programming algorithm for solving the problem. They viewed the problem as a longest path minimisation problem in a PERT/CPM-like network and alternatively as a shortest path problem with side constraints.

Recently, Ho and Ji (2006) employed a HGA (that was proposed in Ho and Ji 2003) to simultaneously solve the component pick-and-place sequencing, feeder setup and component retrieval problem for turret-type SMD placement machines. The component retrieval problem is solved using a nearest neighbour heuristic which aims to minimise the movement of the feeder carrier. Results show that the approach is capable of producing a better solution when there are feeder duplications.

Only a few researchers have focussed on the component retrieval plan problem. The problem might be indirectly solved while determining the component pick-and-place sequence. For example, if the component pick-and-place sequence operation has been determined, the decision of from which feeder slot the appropriate component type could be retrieved (assuming feeder duplication) might also be solved by just selecting the component from the closest feeder slot, which holds the correct component type. Moreover, since one component type can have more than one type of packaging and each packaging type is associated with a certain nozzle type, the problem of determining which feeder slot the appropriate component type could be retrieved from is indirectly solved when determining the nozzle sequencing operation (for the case of having feeder duplication with different types of component packaging). As far as we are aware, no researchers have tried to model this problem as any of the classical optimisation problem such as bin packing, TSP, etc.

\section{Conclusions}

This article has presented a survey of single SMD placement machine optimisation methods. By combining the sub-problems addressed by Crama et al. (2002) and Magyar et al. (1999), this work classified the single SMD placement machine problem into five subproblems. These were: motion control, nozzle 
optimisation, component pick-and-place sequencing, feeder setup and component retrieval planning. These sub-problems are tightly intertwined. Consequently, some researchers solved the problem in an iterative manner, instead of a one-pass procedure through each of the sub-problems and some used an integrated approach, a hierarchical problem approach or just solved the sub-problems independently by making assumptions about the rest of the sub-problems.

As far as we are aware, there have not been many researchers who have reported improving the motion control. This might be because many SMD placement machine use fixed pick-and-place points since not many of them have moveable head $(\mathrm{X}-\mathrm{Y})$, feeder carriers and PCB table.

Many papers define the component pick-andplace optimisation as finding a shortest route to pickand-place the electronic components onto the PCB. This is only true if other factors such as nozzle switching, feeder transportation time (i.e. time taken by the feeder to transport the component to the pickup position), gang pickups (i.e. simultaneous pickup), etc. are ignored. It is more precise to define the component pick-and-place optimisation as finding a shortest time to pick-and-place the components onto the PCB.

Generally, most researchers modelled the component pick-and-place optimisation as a TSP problem. The PCB points are defined as cities whilst the time between placement points represents the distance among cities. Unfortunately, the time between component placement relies on many factors such as nozzle changes, component feeder transportation, the acceleration force on the pre-mounted component (for the case of a movable PCB table), components groupings in a sub-tour (for the case of the machine that has many pipette(s)/nozzle(s) on a head), simultaneous pickups, etc. Indeed, due to technological advancement, the component density on a PCB has gradually increased. Therefore, minimising robot travelling distance/time is becoming less significant and the problem can be redefined as a component pick-and-place optimisation problem, which aims to find the shortest time to pick-and-place electronic components onto the $\mathrm{PCB}$, taking into consideration various factors, such as nozzle changes, simultaneous pickup, feeder transportation time, etc. Since the optimisation of the pick-and-place operations is machine dependent, no general formulation can be defined for optimising the pick-and-place operations. However, one general formulation can be derived for each machine type as classified in Ayob and Kendall (2008).

Evaluating solution quality of the feeder setup is far from easy as other factors have to be considered (e.g. nozzle assignment and sequencing, pick-and-place sequencing, gantry scheduling, etc.). Many previous works have assumed that the other problems were already solved, by fixing the component pick-and-place sequence and solving the feeder setup as a quadratic assignment problem. Few works have focussed on solving the component retrieval plan problem. The problem might be indirectly solved while determining the component pick-and-place sequence.

A nozzle optimisation, in the context of single machine optimisation, can be defined as searching for an effective assignment of nozzles to pipettes that can lead to improving the machine throughput. Good nozzle assignments might lead to having more simultaneous pickup operations; a lesser number of nozzle changes; better movement of the feeder carrier(s), robot arm and/or PCB table, etc. This can ultimately improve the machine throughput. Optimising the pickand-place operations without considering the nozzle change operations may not be efficient, since it may cause many unnecessary nozzle changes that will significantly reduce the machine throughput. As a nozzle change operation is very time consuming, nozzle optimisation can be considered as a crucial factor when improving the machine throughput. Unfortunately, very little work has addressed nozzle optimisation. Since the problem of minimising nozzle switching and minimising the pick-and-place operations are tightly intertwined and should not be solved independently, we strongly recommend that future researchers include the cost of nozzle changes in the objective function of minimising the assembly cycle time.

Since a platform change can take several seconds, optimising this aspect is a key operation. As far as we are aware, this is an unexplored research problem. with many researchers ignoring this aspect and assuming that the tray(s) are always available. Therefore, one further research direction we would encourage is to address the tray feeder problem.

\section{References}

Ahmadi, J., Ahmadi, R., Matsuo, H., and Tirupati, D. (1995), 'Component Fixture Positioning for Printed Circuit Board Assembly with Concurrent Operations', Operations Research, 43, 444-457.

Ahmadi, J., Grotzinger, S., and Johnson, D. (1988), 'Component Allocation and Partitioning for a Dual Delivery Placement Machine', Operations Research, 36, 176-191.

Ahmadi, J., Grotzinger, S., and Johnson, D. (1991), 'Emulating Concurrency in a Circuit Card Assembly System', International Journal of Flexible Manufacturing Systems, 3(1), 45-70. 
Ahmadi, R.H. (1993), 'A Hierarchical Approach to Design, Planning, and Control Problems in Electronic Circuit Card Manufacturing', in Perspectives in Operations Management, ed. R.K. Sarin, Dordrecht: Kluwer Academic Publishers, pp. 409-429.

Ahmadi, R.H., and Mamer, J.W. (1999), 'Routing Heuristics for Automated Pick and Place Machines', European Journal of Operational Research, 117, 533-552.

Altinkemer, K., Kazaz, B., Koksalan, M., and Moskowitz, H. (2000), 'Optimization of Printed Circuit Board Manufacturing: Integrated Modeling and Algorithms', European Journal of Operational Research, 124, 409-421.

Ayob, M., and Kendall, G. (2002a), 'Optimisation for Surface Mount Placement Machines', in Proceedings of the IEEE ICIT'02, Bangkok, 11-14 December 2002, pp. 498-503.

Ayob, M., and Kendall, G. (2002b), 'A New Dynamic Point Specification Approach to Optimise Surface Mount Placement Machine in Printed Circuit Board Assembly', in Proceedings of the IEEE ICIT'02, Bangkok, 11-14 December, pp. 486-491.

Ayob, M., and Kendall, G. (2004). A Nozzle Selection Heuristic to Optimise the Hybrid Pick and Place Machine', in Proceedings of the IEEE Conference on Cybernetics and Intelligent Systems (CIS 2004), Singapore, 1259-1264.

Ayob, M., and Kendall, G. (2005), 'A Triple Objective Function with a Chebychev Dynamic Point Specification Approach to Optimise the SMD Placement Machine', European Journal of Operational Research, 164, 609-626.

Ayob, M., and Kendall, G. (2008), 'A Survey of Surface Mount Device Placement Machine Optimisation: Machine Classification', European Journal of Operational Research, 186(3), 893-914.

Ball, M.O., and Magazine, M.J. (1988), 'Sequencing of Insertions in Printed Circuit Board Assembly', Operations Research, 36, 192-201.

Bard, J.F. (1988), 'A Heuristic for Minimizing the Number of Tool Switches on a Flexible Machine', IIE Transactions, 20, 382-391.

Bard, J.F., Clayton, R.W., and Feo, T.A. (1994), 'Machine Setup and Component Placement in Printed Circuit Board Assembly', International Journal of Flexible Manufacturing Systems, 6, 5-31.

Bentzen, B. (2000). SMD Placement, in the SMT in FOCUS. url:http://www.smtinfocus.com/PDF/SMD_placement.pdf (accessed 25 September, 2002).

Bonert, M., Shu, L.H., and Benhabib, B. (2000), 'Motion Planning for Multi-Robot Assembly Systems', International Journal of Computer Integrated Manufacturing, 13(4), 301-310.

Burke, E.K., Cowling, P., and Keuthen, R. (2001), 'The Printed Circuit Board Assembly Problem: Heuristic Approaches for Multi-headed Placement Machinery', in Proceedings of the IC-AI2001, Las Vegas, CSREA Press, pp. 1456-1462.

Chan, D. (1993), 'Precedence Constrained TSP Applied to Circuit Board Assembly and no Wait Flow-shop', International Journal of Production Research, 31(9), 2171-2177.
Chan, D., and Mercier, D. (1989), 'IC Insertion: An Application of the Traveling Salesman Problem', International Journal of Production Research, 27, 1837-1841.

Chang, T.C., and Terwilliger, J. (1987), 'Rule-based System for Printed Wiring Assembly Process Planning', International Journal of Production Research, 25, 1465-1482.

Chen, W.-S., and Chyu, C.-C. (2003), 'A Minimum Setup Strategy for Sequencing PCBs with Multi-slot Feeders', Integrated Manufacturing Systems, 14(3), 255-267.

Chiu, C., Yih, Y., and Chang, T.C. (1991), 'A Collision-free Sequencing Algorithm for PWB Assembly', Journal of Electronics Manufacturing, 1(1), 1-12.

Chyu, C.-C., and Chang, W.-S. (2008), 'A Genetic Algorithm for the Operational Sequence of a High Speed Chip Placement Machine', International Journal of Manufacturing Technology, 36(9-10), 918-926.

Crama, Y., Flippo, O.E., van de Klundert, J.J., and Spieksma, F.C.R. (1996), 'The Component Retrieval Problem in Printed Circuit Board Assembly', International Journal of Flexible Manufacturing Systems, 8, 287-312.

Crama, Y., Flippo, O.E., Klundert, J.J.V.D., and Spieksma, F.C.R. (1997), 'The Assembly of Printed Circuit Boards: A Case with Multiple Machines and Multiple Board Types', European Journal of Operational Research, 98, 457-472.

Crama, Y., Klundert, J., van de, M., and Spieksma, F.C.R. (2002), 'Production Planning Problems in Printed Circuit Board Assembly', Discrete Applied Mathematics, 123, 339-361.

Crama, Y., Kolen, A.W.J., Oerlemans, A.G., and Spieksma, F.C.R. (1990), 'Throughput Rate Optimization in the Automated Assembly of Printed Circuit Boards', Annals of Operations Research, 26, 455-480.

Crama, Y., Kolen, A.W.J., Oerlemans, A.G., and Spieksma, F.C.R. (1994), 'Minimizing the Number of Tool Switches on a Flexible Machine', International Journal of Flexible Manufacturing Systems, 6, 33-54.

Csaszar, P., Nelson, P.C., Rajbhandari, R.R., and Tirpak, T.M. (2000a), 'Optimization of Automated High-speed Modular Placement Machines Using Knowledge-based Systems', IEEE Transactions on System, Man, and Cybernetics-Part C: Applications and Reviews, 30(4), 408-417.

Csaszar, P., Tirpak, T.M., and Nelson, P.C. (2000b), 'Optimization of a High-speed Placement Machine Using Tabu Search Algorithms', Annals of Operations Research, 96, 125-147.

De Souza, R., and Lijun, W. (1994), 'CPS: A Productivity Tool for Component Placement in Multi-head Concurrent Operation PCBA Machines', Journal of Electronics Manufacturing, 4(2), 71-79.

De Souza, R., and Lijun, W. (1995), 'Intelligent Optimization of Component Insertion in Multi-head Concurrent Operation PCBA Machines', Journal of Intelligent Manufacturing, 6, 235-243.

DePuy, G.W., Ammons, J.C., and McGinnis, L.F. (2000), 'Multiple Assignment of Component Types to Feeder Slots on Automated Printed Circuit Card Placement Machines', 
IEEE Transactions on Electronics Packaging Manufacturing, 23(3), 157-164.

Dikos, A., Nelson, P.C., Tirpak, T.M., and Wang, W. (1997), 'Optimization of High-mix Printed Circuit Card Assembly Using Genetic Algorithms', Annals of Operations Research, 75, 303-324.

Dima SMT Systems. (2003), Hybrid P\&P HP-110, User Manual Version 1.2.

Drezner, Z., and Nof, S. (1984), 'On Optimizing Bin Picking and Insertion Plans for Assembly Robots', IIE Transactions, 16, 262-270.

Duman, E., and Or, I. (2004), 'Precedence Constrained TSP Arising in Printed Circuit Board Assembly', International Journal of Production Research, 42(1), 67-78.

Duman, E., and Or, I. (2007), 'The Quadratic Assignment Problem in the Context of the Printed Circuit Board Assembly Process', Computers \& Operations Research, 34, 163-179.

Ellis, K.P., Vittes, F.J., and Kobza, J.E. (2001), 'Optimizing the Performance of a Surface Mount Placement Machine', IEEE Transactions on Electronic Packaging Manufacturing, 24(3), 160-170.

Foulds, L.R., and Hamacher, H.W. (1993), 'Optimal Bin Location and Sequencing in Printed Circuit Board Assembly', European Journal of Operational Research, 66, 279-290.

Francis, R.L., Hamacher, H.W., Lee, C.-Y., and Yeralan, S. (1994), 'Finding Placement Sequences and Bin Locations for Cartesian Robots', IIE Transactions, 26, 47-59.

Francis, R.L., McGinnis, L.F., and White, J.A. (1992), Facility Layout and Location: An Analytical Approach, Englewood Cliffs, NJ: Prentice-Hall.

Fu, H.-P., and Su, C.-T. (2000), 'A Comparison of Search Techniques for Minimizing Assembly Time in Printed Wiring Assembly', Journal of Production Economics, 63, 83-98.

Gastel, S.V. (2002). A Comparison of SMD Placement Machine Concepts, in the SMT in FOCUS. url:http:// www.smtinfocus.com/PDF/SMD_machine_concept_Asse mbleon.pdf (accessed 6 October, 2004).

Gavish, B., and Seidmann, A. (1988), 'Printed Circuit Boards Assembly Automation-Formulations and Algorithms', in Recent Developments in Production Research, ed. A. Mital, Amsterdam: Elsevier Science, pp. 624-635.

Grotzinger, S. (1992), 'Feeder Assignment Models for Concurrent Placement Machines', IIE Transactions, 24, 31-46.

Grunow, M., Günther, H.-O., Schleusener, M., and Yilmaz, I.O. (2004), 'Operations Planning for Collect-and-Place Machines in PCB Assembly', Computers \& Industrial Engineering, 47(4), 409-429.

Ho, W., and Ji, P. (2003), 'Component Scheduling for Chip Shooter Machines: A Hybrid Genetic Algorithm Approach', Computers and Operations Research, 30, 2175-2189.

Ho, W., and Ji, P. (2004), 'A Hybrid Genetic Algorithm for Component Sequencing and Feeder Arrangement', Journal of Intelligent Manufacturing, 15(3), 307-315.

Ho, W., and Ji, P. (2006), 'A Genetic Algorithm Approach to Optimise Component Placement and Retrieval
Sequence for Chip Shooter Machines', International Journal of Advanced Manufacturing Technology, 28(5-6), $556-560$.

Hop, N.V., and Tabucanon, M.T. (2001a), 'Multiple Criteria Approach for Solving Feeder Assignment and Assembly Sequence Problem in PCB Assembly', Journal of Production Planning and Control, 12(8), 736-744.

Hop, N.V., and Tabucanon, M.T. (2001b), 'Extended Dynamic Point Specification Approach to Sequencing Robot Moves for PCB Assembly', International Journal of Production Research, 39(8), 1671-1687.

Huang, Y.W., Adriance, T., Westby, G., and Srihari, K. (1994), 'A Solution Methodology for the Multiple Batch Surface PCB Placement Sequence Problem', Journal of Electronic Packaging, 116(4), 282-289.

Jeevan, K., Parthiban, A., Seetharamu, K.N., Azid, I.A., and Quadir, G.A. (2002), 'Optimization of PCB Component Placement using Genetic Algorithms', Journal of Electronics Manufacturing, 11(1), 69-79.

Ji, Z., Leu, M.C., and Wong, H. (1992), 'Application of Linear Assignment Model for Planning of Robotic PC Board Assembly', ASME Journal of Electronic Packaging, 114, 455-460.

Ji, P., and Wan, Y.F. (2001), 'Planning for Printed Circuit Board Assembly: The State-of-Art Review', International Journal of Computer Applications in Technology, 14, $136-144$.

Ji, P., Wong, Y.S., Loh, H.T., and Lee, L.C. (1994), 'SMT Production Scheduling: A Generalized Transportation Approach', International Journal of Production Research, 32(10), 2323-2333.

Kang, M.-J. \& Han, C.-G. (1998), 'Solving the Rural Postman Problem using a Genetic Algorithm with a Graph Transformation', in Proceedings of the ACM symposium on Applied Computing, Georgia, USA, February, pp. 356-360.

Kazaz, B., and Altinkemer, K. (2003), 'Optimization of Multi-feeder (depot) Printed Circuit Board Manufacturing with Error Guarantees', European Journal of Operational Research, 150(2), 370-394.

Khoo, L.P., and Loh, K.M. (2000), 'A Genetic Algorithms Enhanced Planning System for Surface Mount PCB Assembly', International Journal of Advanced Manufacturing Technology, 16(4), 289-296.

Khoo, L.P., and Ng, T.K. (1998), 'A Genetic Algorithmbased Planning System for PCB Component Placement', International Journal of Production Economics, 54, 321-332.

Khoo, L.P., and Ong, N.S. (1998), 'PCB Assembly Planning using Genetic Algorithms. International', Journal of Advanced Manufacturing Technology, 14, 363-368.

Klincewicz, J.G., and Rajan, A. (1994), 'Using GRASP to Solve the Component Grouping Problem', Naval Research Logistics, 41, 893-912.

Klomp, C., Klundert, J.J.V.D., Spieksma, F.C.R., and Voogt, S. (2000), 'The Feeder Rack Assignment Problem in PCB Assembly: A Case Study', International Journal of Production Economics, 64, 399-407. 
Kumar, R., and Luo, Z. (2003), 'Optimizing the Operation Sequence of a Chip Placement Machine using TSP Model', IEEE Transactions on Electronics Packaging Manufacturing, 26(1), 14-21.

Lee, S.H., Lee, B.H., and Park, T.H. (1999), 'A Hierarchical Method to Improve the Productivity of a Multihead Surface Mounting Machine', in Proceedings of the 1999 IEEE on Robotics and Automation, Michigan, pp. 2110-2115.

Leipälä, T., and Nevalainen, O. (1989), 'Optimization of the Movements of a Component Placement Machine', European Journal of Operational Research, 38, 167-177.

Leu, M.C., Wong, H., and Ji, Z. (1993), 'Planning of Component Placement/Insertion Sequence and Feeder Setup in PCB Assembly using Genetic Algorithm', Journal of Electronic Packaging, 115, 424-432.

Li, S. Hu, C. and Tian, F. (2008). Enhancing Optimal Feeder Assignment of the Multi-head Surface Mounting Machine using Genetic Algorithms. Applied Soft Computing, 8(1), 522-529.

Liggett, R.S. (1981), 'The Quadratic Assignment Problem: An Experimental Investigation of Solution Strategies', Management Science, 27(4), 442-458.

Loh, T.S, Bukkapatnam, S.T.S., Medeiros, D., and Kwon, H.A. (2001), 'Genetic Algorithm for Sequential Part Assignment for PCB Assembly', Computers and Industrial Engineering, 40, 293-307.

Magyar, G., Johnsson, M., and Nevalainen, O. (1999), 'On Solving Single Machine Optimization Problems in Electronics Assembly', Journal of Electronics Manufacturing, 9(4), 249-267.

McGinnis, L.F., Ammons, J.C., Carlyle, M., Cranmer, L., Depuy, G.W., Ellis, K.P., Tovey, C.A., and Xu, H. (1992), 'Automated Process Planning for Printed Circuit Card Assembly', IIE Transactions: Scheduling \& Logistics, 24, 18-30.

Mettalla, E.G., and Egeblu, P.J. (1989), 'Alternative Approaches to Sequencing Robot Moves for PCB Assembly', International Journal of Computer Integrated Manufacturing, 2, 243-256.

Moyer, L.K., and Gupta, S.M. (1996a), 'SMT Feeder Slot Assignment for Predetermined Component Placement Paths', Journal of Electronics Manufacturing, 6, 173-192.

Moyer, L.K., and Gupta, S.M. (1996b), 'Simultaneous Component Sequencing and Feeder Assignment for High Speed Chip Shooter Machines', Journal of Electronics Manufacturing, 6, 271-305.

Moyer, L.K., and Gupta, S.M. (1997), 'An Efficient Assembly Sequencing Heuristic for Printed Circuit Board Configurations', Journal of Electronics Manufacturing, 7, $143-160$

Nelson, K.M. \& Wille, L.T. (1995), 'Comparative Study of Heuristics for Optimal Printed Circuit Board Assembly', in Proceedings of Southcon'95, Fort Lauderdale, Florida, USA, pp. 322-327.

Ng, M. (1998), 'Heuristics Approach to Printed Circuit Board Insertion Problem', Journal of the Operational Resarch Society, 49, 1051-1059.
Ohno, K., Jin, Z., and Elmaghraby, S.E. (1999), 'An Optimal Assembly mode of Multi-type Printed Circuit Boards', Computers \& Industrial Engineering, 36, 451-471.

Ong, N.-S., and Khoo, L.P. (1999), 'Genetic Algorithm Approach in PCB Assembly', Integrated Manufacturing Systems, 10(5), 256-265.

Ong, N.S., and Tan, W.C. (2002), 'Sequence Placement Planning for High-speed PCB Assembly Machine', Integrated Manufacturing Systems, 13(1), 35-45.

Pearn, W.L., and Wu, T.C. (1995), 'Algorithm for the Rural Postman Problem', Computers and Operations Research, 22(8), 819-828.

Sadiq, M., Landers, L., and Don Taylor, G. (1993), 'A Heuristic Algorithm for Minimizing Total Production Time for a Sequence of Jobs on a Surface Mount Placement Machine', International Journal of Production Research, 31(6), 1327-1341.

Safai, F. (1996), 'Cycle Time Improvement for Fuji IP2 Pick-and-Place Machines', Hewlett-Packard Journal, 47(4), 80-86.

Sanchez, J.M., and Priest, J.W. (1991), 'Optimal Component Insertion Sequence Planning Methodology for the Semiautomatic Assembly PCB', Journal of Intelligent Manufacturing, 2, 177-188.

Shih, W., Srihari, K., and Adriance, J. (1996), 'Expert System Based Placement Sequence Identification for Surface Mount PCB Assembly', International Journal of Advanced Manufacturing Technology, 11, 413-424.

Sohn, J., and Park, S. (1996), 'Efficient Operation of a Surface Mounting Machine with a Multihead Turret', International Journal of Production Research, 34(4), 1131-1143.

Su, C., and Fu, H. (1998), 'A Simulated Annealing Heuristic for Robotics Assembly using the Dynamic Pick-and-Place Model', Production Planning and Control, 9, 795-802.

Su, C., Ho, L., and Fu, H. (1998), 'A Novel Tabu Search Approach to find the Best Placement Sequence and Magazine Assignment in Dynamic Robotics Assembly', Integrated Manufacturing Systems, 9, 366-376.

Su, Y., and Srihari, K. (1996), 'Placement Sequence Identification using Artificial Neural Networks in Surface Mount PCB Assembly', International Journal of Advanced Manufacturing Technology, 11, 285-299.

Su, Y.-C., Wang, C., Egbelu, P.J., and Cannon, D.J. (1995), 'A Dynamic Point Specification Approach to Sequencing Robot Moves for PCB Assembly', International Journal of Computer integrated Manufacturing, 8(6), 448-456.

Sun, D.-S., Lee, T.-E. \& Kim, K.-H. (2005). 'Component Allocation and Feeder Arrangement for a Dual-gantry Multi-head Surface Mounting Placement Tool', International Journal of Production Economics, 95(2), 245-264.

Tirpak, T.M. (2000), 'Design to Manufacturing Information Management for Electronics Assembly', International Journal of Flexible Manufacturing Systems, 12, 189-205.

Tirpak, T.M., Nelson, P.C., and Aswani, A.J. (2000), 'Optimization of Revolver Head SMT Machines using Adaptive Simulated Annealing (ASA)', Electronics Manufacturing Technology Sympoium, Twenty-Sixth IEEE/CPMT, IEEE Press, pp. 214-220. 
Tsai, T.-N., Yang, T., and Hou, P.-A. (2005), 'Neurofuzzy Modelling of the Reflow Thermal Profile for Surface Mount Assembly', International Journal of Systems Science, 36(2), 89-101.

Van Breedam, A. (2001), 'Comparing Descent Heuristics and Metaheuristics for the Vehicle Routing Problem', Computers \& Operations Research, 28, 289-315.

Van Laarhoven, P.J.M., and Zijm, W.H.M. (1993), 'Production Preparation and Numerical Control in PCB Assembly', International Journal of Flexible Manufacturing Systems, 5, 187-207.

Wang, C. (1996), 'Layout Designs for Robotic PCB Assembly', Journal of Integrated Manufacturing Systems, 7(4), 39-52.

Wang, C., Ho, L.S., Fu, H.P., and Su, Y.C. (1995). A Magazine Assignment Heuristic for Robotic Assembly using the Dynamic Pick-and-Place Approach. Report No. IM84J05, Department of Industrial Management, ChungHua Polytechnic Institute, Hsinchu, Taiwan.
Wang, C., Ho, L.S., Fu, H.P, and Su, Y.C. (1997), 'A Magazine Assignment Heuristic for Robotic Assembly using the Dynamic Pick and Place Approach', International Journal of Industrial Engineering, 4(1), 24-33.

Wang, C., Ho, L.-S., and Cannon, D.J. (1998), 'Heuristics for assembly Sequencing and Relative Magazine Assignment for Robotic Assembly', Computers \& Industrial Engineering, 34, 423-431.

Wang, W., Nelson, P.C., and Tirpak, T.M. (1999), 'Optimization of High-speed Multistation SMT Placement Machines using Evolutionary Algorithms', IEEE Transactions on Electronics Packaging Manufacturing, 22(2), 137-146.

Wong, H., and Leu, M.C. (1993), 'Adaptive Genetic Algorithm for Optimal Printed Circuit Board Assembly Planning', Annals CIRP, 42(1), 17-20.

Yeo, S.H., Low, C.W., and Yong, K.H. (1996), 'A Rulebased Frame System for Concurrent Assembly Machines', International Journal of Advanced Manufacturing Technology, 12, 370-376. 